\title{
An Efficient Way to Recognize a Person in Surveillance Video
}

\author{
Sowmiya $\mathrm{V}^{\mathrm{a}, 1}$ and Malathi $\mathrm{S}^{\mathrm{b}}$ \\ ${ }^{a} P G$ scholar ,Dept of CSE,Panimalar engineering College, Chennai \\ ${ }^{b}$ Professor,Dept of CSE, Panimalar engineering college, Chennai
}

\begin{abstract}
This paper is presented for tracking a person in the visual surveillance system and to detect the pose of that particular person presented in that video. This concept is fully based on monitoring elderly people, patients, and people with disabilities. In the present day monitoring them for the whole day has been difficult and high-end surveillance is also difficult. So automatic pose detection makes these tasks easier. The algorithm presented here not only recognize human pose like running, walking but also includes abnormal pose detection like fall detection for elderly people, people with chronic disease are suffering from strokes and often fell down, the algorithm can also detect the various pose of a human. Although the technique to find human pose are existing but detecting human pose in surveillance system are difficult due to its movement presented in the video. In particular, we demonstrated by separating the motion pixel by (MPEM) pixel expectation maximization. (MPEM) is the process of separating the movement pixel. Finally KL transform is applied to track the action and pose of a human. The kinetic sensor SDK used to recognize the human body with high accuracy and more efficiency. Human body and face authentication modality are performed using various methods such as artificial neural network (ANN). Several experiments are performed to validate the effectiveness are performed to validate the effectiveness of the proposed system tracking approach, the result of which seems quite promising.
\end{abstract}

Keywords. kinetic sensor, artificial neural network (ANN), deep neural network.

\section{Introduction}

Human action focus has end up an important topic in computer vision. The system reported here is to recognize human action in an environment for which prior knowledge is handy. These details are obtained by training the system with a lot of datasets. Our system is capable of recognizing people in the surveillance camera. Tracking a person and finding pose of that particular human body in the surveillance camera has been accomplished by means of extracting a characteristic of body components, background picture has to be subtracted and defining the extracted characteristic are the following key factors of identification to actual time monitoring. There are two kinds of strategies that use the region based method first for shifting object detection and the next one is boundary based approach. However the manner of the historical past takes to determine model. The proposed algorithm fetches the feature of human body components. those element are processed by means of motion 
pixel expectation maximization Expectation maximization algorithm can be utilized for latent variable (variable that are not once determined and are actually inferred from the value of the other discovered variable). To calculate their values on the situation that we are conscious of the customary structure of the distribution of chances governing those latent variable. This algorithm is a the sources of many unsupervised clustering algorithm. we trade the $\mathrm{CNN}$ to sort the target location within the object patches.

In earlier days Douglas Ayers, Shah[1] has designed Mubarak a system that qualified to recognize the gesture by using prior understanding about the blueprint of the room. The ideas presented in this system are relevant to self-operated security. The result of this apparatus is both a textual and a key frame elucidation of the recognized motion. According to the modern world by using latest technology. We have designed automatic recognize which can be done without the help of any manual involvement Ismail presented [2] a paper with the concept of hydra technology which is capable of signifying the existence of two people. Then they are shown by a big scale with $320 * 240$ resolutions. The estimation model employed by Hydra to track multiple people before, during and after occlusion. The technology used here Hydra (processor is a hardware accelerated signal processing library written with CUDA).

In [3] Alan J. Lipton et al. shows system strongly found targets of interest, omit background clutter; and frequently tracks over a long distances and with the long periods of time regardless of occlusions, appearance changes and cessation of target motion for this correlation matching, temporal steadiness differencing are used . Using a combination of domain information and temporal, targets are robustly identified in spite of one sided occlusions and ambiguous poses, and Background clutter is effectively removed. Koichi Sato and J. K. Aggarwal In [4] the technique introduced here for recognition Two person communicating in outdoor.This technique capable of recognizing nine different communication at a given time[5]. The accuracy found to be $86 \%$ with the solid advantages of robustness with degree of accuracy.

\section{Tracking Human in Surveillance}

Activity recognition is an important problem in smart video surveillance and it is the basic problem in computer vision. To defeat this difficult there have been many pieces of research undergoing in the name as human tracking. In the presented paper we have used convolution neural network to train the system for automatically detecting the human in surveillance[8-9].

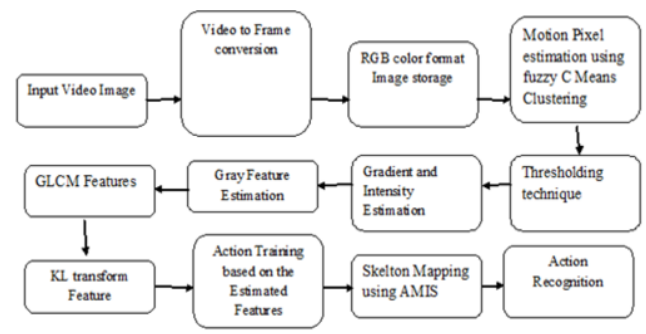

Input video is acquired from the surveillance database .video acquisition using frame converser system is used to convert a video frame into a picture, the MATLAB 
function 'frame2im' is employed. Choose Photoshop image sequence select a desired video and finally convert the video into image[6-7].

\section{RGB Color Format Image Storage}

The shade of pixel is chosen by the blend of the red, green and blue forces put away in each shading plane at the pixel's area. Illustrations record design store RGB picture as 24-piece picture, where the red, green and blue parts are 8 bits each.

\section{Motion Pixel Estimation Using Fuzzy C Means Clustering}

Another methodology for dark level picture division is exhibited utilizing a probabilistic fluffy c-implies grouping calculation. This methodology consolidates the spatial probabilistic data and the fluffy enrollment work in the bunching procedure. The proposed probabilistic fluffy c-implies technique can manage picture division in an uproarious situation. Feature Extraction is a method of capturing visual content of images for indexing \& retrieval. This paper presents an application of gray level cooccurrence matrix (GLCM) to extract second order statistical texture features for motion estimation of images. And gray feature extraction in done. A picture angle is a directional change in the force or shading in a picture. The angle of the picture is one of the basic structure obstructs in picture handling. For instance, the canny edge locator utilizes picture angle for edge discovery.

\section{K means Magnitude clustering}
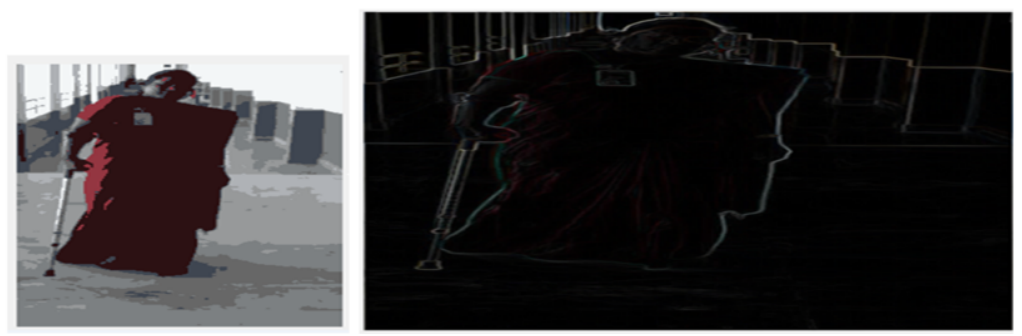

Figure 1. Motion pixel expectation maximization Figure 2. K-means magnitude clustering

Highlight Extraction is a technique for catching visual substance of pictures for ordering and recovery. This paper shows the use of dark level co-event grid (GLCM) to separate second request statistical texture highlights for movement estimation of pictures. A picture inclination is a directional change in the power or shading in a picture. The inclination of the picture is one of the key structure obstructs in picture preparing. For instance, the Canny edge identifier utilizes a picture slope for edge identification. Image Thresholding is a simple, yet effective, way of segregation an image into a foreground and background. This image analysis technique is a type of image segmentation that detach objects by transform grayscale images into binary images. Image Thresholding is most productive in images with high levels of variation. 


\section{High Frequency Coefficients}

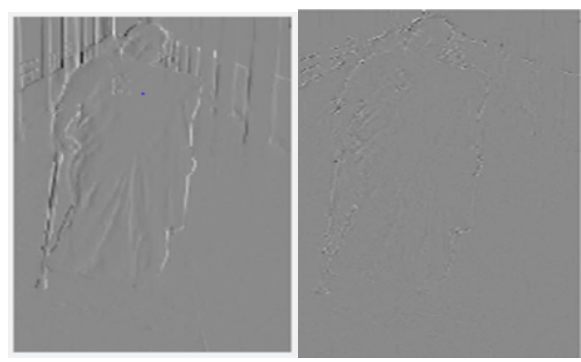

Figure 3. High and low frequency coefficient

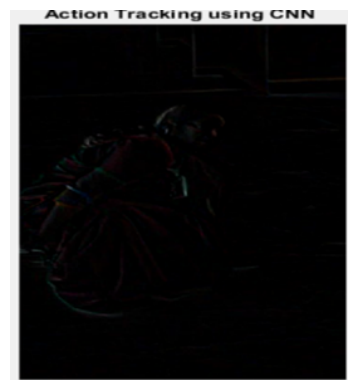

Figure 4. Pose action tracking

The reinforcement learning agent has to resolve how to accomplishment its perform its task. In the absent of existing training data, the agent acquire knowledge from experience. it collect the training example. Through trial and error as it seek with the goal of maximizing long term pay.

\section{Action Recognition}

To perceive activities of a human appropriately, a calculation must have the option to identify an individual precisely in some random picture. This information will be utilized to find out what activity the client is performing. Numerous strategies have been suggested that utilization a distinction picture procedure in which the picture of the client is subtracted from a recently known picture with a similar foundation, without the client. Early stage phase of improvement along these lines plentiful additional work stays to be done to deal with the proceeding with difficulties. Obviously creating keen calculations for finding the matter of action acknowledgment would yield tremendous potential for a larger than usual assortment of potential applications.

\section{Training Data Set}

A surveillance camera has programmed in a way which a device can automatically recognize human pose using a convolution neural network based on deep neural network. 


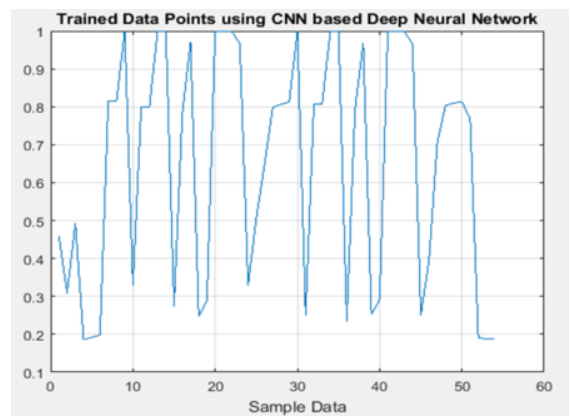

\section{Conclusion}

This paper is presented for tracking a person in the visual surveillance system and to detect the pose of that particular person presented in that video. This concept is fully based on monitoring elderly people, patients, and people with disabilities. So that,KL transform is applied to track the action and pose of a human. The kinetic sensor SDK used to recognize the human body with high accuracy and more efficiency. Human body and face authentication modality are performed using various methods such as artificial neural network (ANN). Several experiments are performed to validate the effectiveness are performed to validate the effectiveness of the proposed system tracking approach, the result of which seems quite promising.

\section{References}

[1] D. Ayers and M. Shah, Recognizing human actions in a static room. in Proceedings Fourth IEEE Workshop on Applications of Computer Vision. WACV'98 (Cat. No. 98EX201). IEEE, 1998, pp. 4247.

[2] I. Haritaoglu, Multiple people detection and tracking using silhouettes. in Second IEEE Workshop Visual Surveillance. 1999, 1999.

[3] A. J. Lipton, H. Fujiyoshi, and R. S. Patil, Moving target classification and tracking from real-time video. in Proceedings Fourth IEEE Workshop on Applications of Computer Vision. WACV'98 (Cat. No. 98EX201). IEEE, 1998, pp. 8-14.

[4] K. Sato and J. Aggarwal, Tracking and recognizing two-person interactions in outdoor image sequences. in Proceedings 2001 IEEE Workshop on Multi-Object Tracking. IEEE, 2001, pp. 87-94.

[5] C.-M. Huang, Y.-R. Chen, and L.-C. Fu, Visual tracking of human head and arms using adaptive multiple importance sampling on a single camera in cluttered environments.IEEE Sensors Journal, vol. 14, no. 7, pp. 2267-2275, 2014.

[6] R. Polana and R. Nelson, Low level recognition of human motion (or how to get your man without finding his body parts). in Proceedings of 1994 IEEE Workshop on Motion of Non-rigid and Articulated Objects. IEEE, 1994, pp. 77-82.

[7] M. Yin, Y. Bo, G. Zhao, and W. Zou, Adaptive block-fusion multiple feature tracking in a particle filter framework. in 2013 IEEE International Conference.

[8] V.D.Ambeth Kumar, V.D.Ashok Kumar, S.Malathi, K.Vengatesan, M.Ramakrishnan, "FACIAL RECOGNITION SYSTEM FOR SUSPECT IDENTIFICATION USING A SURVEILLANCE CAMERA", Pattern Recognition and Image Analysis (Springer), Volume 28, Issue 3, pp 410-420, 2018.

[9] V.D.Ambeth Kumar, G.Saranya , D.Elangovan, V.RahulChiranjeevi, V.D.Ashok Kumar, "IOT Based Smart Museum Using Wearable Device”, Lecture Notes in Networks and Systems, Vol.55, pp: 33-42, 2018 